\title{
Lessons Learned an der DIU
}

\author{
S. Richter \\ Digital Managerin der DIU Dresden International University
}

\begin{abstract}
Es ist notwendig, ein Umdenken im Bereich der Bildung zu erreichen - nicht zuletzt aufgrund der permanenten und schnellen Veränderungen, die durch Technologie möglich geworden sind. Die Digitalisierung ist dabei nur ein Teilstück, welches die Anpassungen zum einen begünstigt, zum anderen aber auch erfordert. Wissen ist jederzeit abrufbar. Zu jedem Thema findet man Informationen im Internet. Deshalb ist es zum einen wichtig, Kompetenzen zu entwickeln im Umgang mit der Vielfalt der Daten, aber auch zu erkennen, dass das reine Lehren von Wissen nicht mehr zeitgemäß ist und unsere Kinder und Jugendlichen nicht auf das vorbereitet, womit sie in ihrem Arbeitsleben konfrontiert sein werden. Die Erlangung von Kompetenzen wie Kommunikation, Kreativität, kritisches Denken und Kollaboration (4 K) rückt in den Vordergrund und löst reinen Wissenserwerb ab. Wie können wir nun mit Hilfe von Tools und Methoden ein neues Zeitalter der Bildung zumindest einmal einläuten? Wie können wir erreichen, dass Digitalität als Unterstützung und nicht als in der Pandemie notwendiges aber vorübergehendes Übel betrachtet wird? Welche Ideen haben wir für hybride Lernsettings entwickelt? Wir haben seit März 2020 sehr viel lernen dürfen und einiges ausprobiert und möchten unsere Learnings im folgenden Bericht teilen.
\end{abstract}

It is essential to rethink in the field of education - not least because of the permanent and rapid changes that have become possible through technology. Digitalisation is only one part of this, which on the one hand favours the adjustments, but on the other hand also requires them. Knowledge can be accessed at any time. Information on every topic can be found on the internet. Therefore, it is important to develop competences in dealing with the diversity of data, but also to recognise that the mere teaching of knowledge is no longer up-to-date and does not prepare our children and young people for what they will be confronted with in their working lives. The acquisition of competences such as communication, creativity, critical thinking and collaboration (4Cs) move to the front and replace pure knowledge acquisition. Therefore, the question arises, how we can use tools and methods to usher in a new age of education. How can we achieve that digitality is seen as a support and not as a necessary but temporary evil in the pandemic? What ideas have we developed for hybrid learning settings? Since March 2020, we have been able to learn a lot and try out a few things and would like to share our learnings in the following report.

*Corresponding author: sandra.richter@di-uni.de 


\section{Unsere Ausgangssituation}

Die Ausgangssituation an der DIU ist etwas anders als an der Technischen Universität Dresden, da wir als private Universität mit freien Dozent:innen arbeiten, die häufig nur einen Kurs pro Studiengang mit etwa 20 Unterrichtseinheiten oder weniger durchführen. Der folgende Erfahrungsbericht setzt sich deshalb allgemein mit unseren Learnings im Hinblick auf digitales bzw. hybrides Lernen auseinander und zeigt eins mehr als deutlich: Nur eine Plattform zur Verfügung zu stellen, um dort Präsentationen digital vortragen zu lassen, genügt bei weitem nicht.

Virtuell ist die Gefahr noch größer, abgelenkt zu werden, wenn die Lehrveranstaltung nicht interessant genug ist. Da man eben sowieso am PC sitzt, kann man auch schnell die ankommende Mail bearbeiten oder sich um sein Twitter-Profil kümmern. Mit einem Klick sind die Teilnehmenden dabei oder eben wieder weg. Das heißt, dass es wichtig ist, spannende Formate zu kreieren, die möglichst interaktiv sind, um die Teilnehmenden mitzunehmen. Deshalb sprechen wir gern von einer Lernreise und nicht von einer Vorlesung.

In der Diskussion geht es aber nicht mehr nur um digital, hybrid oder analog, es geht darum, wie Bildung sowohl im schulischen als auch hochschulischen Kontext aussehen soll.

Für uns steht fest, dass es eine individuellere Betreuung der Studierenden braucht und dass kollaborative Projekte und selbstgesteuertes Lernen zielführender sind als das stete Eintrichtern von Wissen, welches die Studierenden nach der Prüfung direkt wieder vergessen. Um in der heutigen schnelllebigen Zeit die Probleme von morgen lösen zu können, braucht es ein Umdenken im Bildungsbereich und auch ein neues Rollenverständnis. Studierende erhalten mehr Verantwortung für das Erreichen von Lernzielen, Lehrkräfte werden zu Lernbegleiter:innen.

Im Methodenbuch für digitalen Unterricht [1] findet sich folgendes Zitat:

„Eine Bildung, um Probleme zu lösen, die wir aktuell noch gar nicht kennen."
Die folgende Tabelle zeigt, was damit gemeint ist:

\begin{tabular}{|l|l|}
\hline Weg von & Hin zu \\
\hline Wissen & Kompetenz \\
\hline Vermitteln \& Belehren & Herausfinden \\
\hline Analog & Digital \& hybrid \\
\hline Starren Strukturen & $\begin{array}{l}\text { Verknüpften und flexib- } \\
\text { len Bildungskontexten }\end{array}$ \\
\hline Lernen im Gleichtakt & Individuellem Lernen \\
\hline Einzelkämpfertum & Zusammenarbeit \\
\hline (Fremd-)Steuerung & $\begin{array}{l}\text { Selbstorganisation und } \\
\text { Mitbestimmung }\end{array}$ \\
\hline Eindimensionalität & $\begin{array}{l}\text { Mehrperspektivität und } \\
\text { Vernetzung }\end{array}$ \\
\hline Lehrkraftzentrierung & Lernendenzentrierung \\
\hline $\begin{array}{l}\text { Feststehenden Ergebnis- } \\
\text { sen }\end{array}$ & Ergebnisoffenheit \\
\hline Vorgegebener Bedeutung & Persönlichem Sinn \\
\hline Lehrenden & Lernenden \\
\hline
\end{tabular}

Tab. 1: Bildung der Zukunft [1]

Doch wie können wir das erreichen?

In den folgenden Abschnitten haben wir einige unserer Gedanken und Ideen zusammengetragen, jedoch lernen wir immer wieder Neues dazu. Deshalb ist aus unserer Sicht der erste Schritt im Hinblick auf ein Umdenken im Bildungsbereich, dass sich Lehrkräfte ebenfalls als Lernende verstehen und in den gemeinsamen offenen und vertrauensvollen Austausch kommen, um Wissen zu teilen, gemeinsam Lernkonzepte zu erarbeiten und das bisherige Einzelkämpfer:innendasein aufzugeben.

Die DIU bietet hierfür regelmäßig den DIUTalk an, hat eine LinkedIn-Community ins Leben gerufen. Darüber hinaus geben wir unser Wissen in kostenfreien Workshops an interessierte Dozierende weiter.

Unser Motto: Wissen teilen ist Kultur!

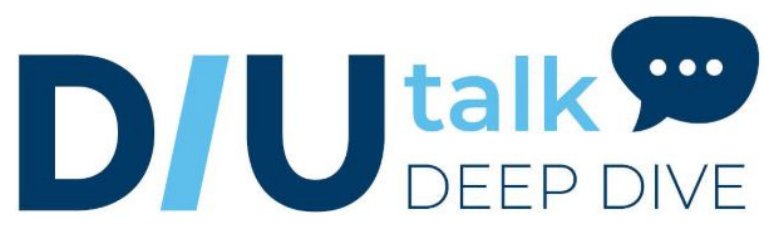

Abb. 1: Logo des Workshopformats der DIU 


\section{Future Skills - die $4 \mathrm{~K}$}

Besonderes Augenmerk im schulischen als auch hochschulischen Kontext sollte auf die 4 $\mathrm{K}$ - Kritisches Denken, Kreativität, Kollaboration und Kommunikation - gelegt werden. Wie erreichen wir, dass unsere Studierenden sich kritisch mit Themen auseinandersetzen, kreative Lösungsansätze kreieren, sich vernetzen, um gemeinsam Herausforderungen zu meistern und klar und wertschätzend kommunizieren, um überhaupt mit Anderen kollaborieren zu können?

Das Verwenden eines digitalen Tools führt nicht zwangsläufig zu einem spannenden Lernszenario. Allein die Digitalisierung ist also nicht ausreichend, um die längst notwendige Veränderung im Bereich Bildung zu bewirken. Vielmehr bedingt die Digitalisierung, dass wir in der Bildung neue Wege einschlagen müssen, um Kompetenzen zu erlangen, die für uns und unsere Studierenden in der heutigen und zukünftigen Welt nützlich und sinnvoll sind. Nicht das Sammeln von Zertifikaten und Abschlüssen oder das Horten von Wissen sind das Ziel, sondern die Lernreise als solche und das tiefe Verständnis für das Lernthema durch die intensive Auseinandersetzung damit, so dass Lernende in der Lage sind, konkrete Fragestellungen zu verstehen und Probleme zu lösen und Andere gezielt um Unterstützung zu bitten.

In den nachfolgenden Abschnitten zeigen wir einige Methoden, Tipps und Hinweise, wie "New Learning" aus unserer Sicht gelingen kann und wie Digitalität uns dabei unterstützt.

\section{Ice-Breaker und Warm-up}

Trifft man auf eine neue Studiengruppe ausschließlich im digitalen Umfeld, gehen einige Informationen verloren, die man im persönlichen Umgang miteinander automatisch wahrnimmt. Es ist demnach erschwert, eine Person virtuell kennenzulernen, weil Bezugspunkte fehlen und natürlich auch die gemeinsam verbrachten Pausen, in denen man sich austauscht.

Doch es ist nicht unmöglich, auch im virtuellen Raum Nähe aufzubauen und sich kennenzulernen.
Es ist hilfreich, mit Warm-up-Übungen zu starten, damit sich alle in lockerer Runde kennenlernen können. Hierfür gibt es zahlreiche Ideensammlungen im Internet z.B. auch unter http://tscheck.in/ oder https://internet quatsch.de/.

Methodisch empfehlen wir „Impromptu Networking" aus dem Liberating-Structures-Werkzeugkoffer. Beim „Impromptu Networking" werden alle Teilnehmenden bestenfalls zufällig und automatisch in Zweiergruppen zusammengebracht, um vier Minuten lang zu einer beliebigen Einstiegsfrage, die durchaus zunächst ganz unabhängig vom Lernfeld sein darf, ins Gespräch zu kommen. Es gibt drei Runden, also drei Fragen. Die Gruppenzusammensetzung wird dreimal neu verteilt, so dass sich möglichst viele Teilnehmende bereits begegnet sind. Die Beschreibungen aller Liberating Structures sind unter https://libera tingstructures.de/ zu finden. Diese 33 Mikrostrukturen helfen, ganz gezielt an Herausforderungen zu arbeiten und zu gewährleisten, dass jede:r einbezogen wird.

Auch das Einbinden von Live-Umfrage-Tools wie Mentimeter https://www.mentime ter.com/ oder https://invote.de/ kann nützlich sein, um einen möglichst lockeren Einstieg zu finden und ein gutes Kennenlernen zu ermöglichen. Persönliche Fragen z.B. nach dem Lieblingsurlaubsland sind sinnvoll, bevor ins Fachliche übergegangen wird. So finden die Studierenden leichter Anknüpfungspunkte und können durch Gemeinsamkeiten Vertrauen zueinander aufbauen. Unsere Überzeugung ist, dass eine gute Bindung auch online entstehen kann, wenn man Raum lässt für ein vertrauensvolles und fröhliches Miteinander.

Natürlich ist damit nicht gemeint, dass man zu viele Spiele spielt und das Lernen darüber vergisst. Eine gute Planung der Eisbrecherphase ist unerlässlich. Das gilt im analogen, hybriden und digitalen Lernraum gleichermaßen. Wenn bestimmte Tools und Methoden genutzt werden sollen, ist genügend Zeit einzuplanen diese in Ruhe einzuführen und zu erläutern. Das ist nicht zu unterschätzen, denn nur wenn alle die Anwendung beherrschen, kann die Zusammenarbeit auch gut funktionieren.

Regeln für Online-Veranstaltungen können 
ebenfalls sehr hilfreich sei. Die DIU hat folgenden Online-Knigge entwickelt, der für Meetings zur Anwendung kommt, aber durchaus auch in Lernveranstaltungen gelten kann:

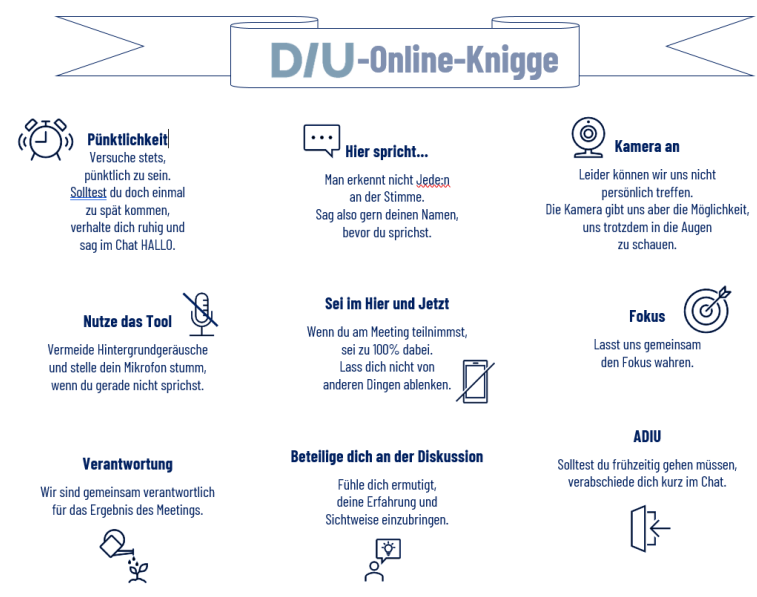

Abb. 2: Online-Knigge der DIU

\section{Kameras an}

In unserem Online-Knigge ist auch das Thema „Kamera" verankert.

Wenn alle Teilnehmenden ihre Kameras ausgeschaltet lassen, ist es schwer für Dozierende, eine Bindung aufzubauen und Feedback zu bekommen. Man hat das Gefühl, gegen eine stille schwarze Wand zu reden und verfällt ins Monologisieren. Oft genügt es, die Teilnehmenden zu bitten, ihre Kamera anzuschalten. Dies sollte nicht vorwurfsvoll formuliert sein, sondern eher mit einem freundlichen Hinweis, dass man sich sehr freuen würde, die Teilnehmenden auch zu sehen. Sollte dies wider Erwarten nicht dazu führen, dass zumindest der Großteil sich zeigt, gibt es einige Tipps.

Es helfen vor allem spielerische Übungen, die das Kamerabild einbeziehen, z.B. kann der Dozierende Ja-/Nein-Fragen stellen und wer mit Ja antwortet, schaltet die Kamera ein und wer mit Nein antwortet, lässt diese zunächst aus. So kann direkt zu Beginn bereits abgefragt werden, welche Themen für die Teilnehmenden besonders interessant oder unbedingt nachzuholen sind und zusätzlich nutzen die Teilnehmenden nun die Kamera. Ein weiteres Spiel für den Einstieg ist es, Gegenstände in die Kamera zu halten und alle Teilnehmenden zu bitten, nun einen ähnlichen Gegenstand zu suchen und ebenfalls zu zeigen. Dies kann sogar echte Bewegung in die Lernveranstaltung hineinbringen und der ein oder andere fröhliche Lacher wird auch zu vernehmen sein.

Eine wunderbare Methode aus dem Improvisationstheater ist, dass die Teilnehmenden nacheinander ihren Namen sagen und dazu eine Geste vollführen. die auch dazu dient, sich die Namen der Anderen zu merken.

Solche spielerischen Ansätze bereits in die Icebreaker-Phase zu planen, ist aus unserer Sicht ein doppelter Gewinn.

\section{Aktivierung der Teilnehmenden}

Bei analogen Veranstaltungen spricht man davon, dass die Aufmerksamkeit nach 20 Minuten nachlässt. In Online-Veranstaltungen ist dies laut Andrea Heitmann in einem Ihrer Workshops zu Digitaler Rhetorik bereits nach 7 Minuten der Fall.

Deshalb ist es wichtig, regelmäßig Interaktionen in die Lernveranstaltung einzubauen. Dies bedeutet nicht, dass man permanent Spiele oder aufwendige Umfragen einbetten soll. Auch kurze Rückfragen, kleinere Abstimmungen o.ä. genügen, um die Aufmerksamkeit zu erhalten oder zurückzugewinnen. Die meisten OnlinePlattformen bieten mit Live-Reaktionen eine gute Möglichkeit, auch zwischendurch Feedback zu geben oder einzuholen. Ein Chatstorm ist ebenfalls eine wunderbare Option. Es wird eine Frage gestellt, die Teilnehmenden bekommen kurz Zeit, ihre Antwort im Chat zu formulieren, klicken aber erst alle auf Kommando zeitgleich auf Absenden. So vermeidet man, dass Ideen kopiert werden.

Nichtsdestotrotz finden spielerische Ansätze mit Challenge-Charakter bei den Studierenden sehr viel Anklang. Hier gibt es viele Tools wie beispielsweise https://kahoot.it/, die man einsetzen kann, um zwischendurch ein Live-Quiz zu generieren. Der spielerische Charakter sollte dennoch immer mit einer kritischen Auseinandersetzung der gestellten Frage einhergehen, wenn man ein Quiz nicht nur zum Spaß, sondern tatsächlich als lernunterstützende Methode einfließen lassen möchte.

Hier einige weitere Beispiele:

https://jeopardylabs.com/, Montagsmaler unter https://skribbl.io/ oder https://stadtlandfluss.cool/. 
Gerade letzteres kann durch die Option, eigene Kategorien einzubeziehen, für Spaß sorgen. Kreativität wird freigesetzt und Schnelligkeit belohnt.

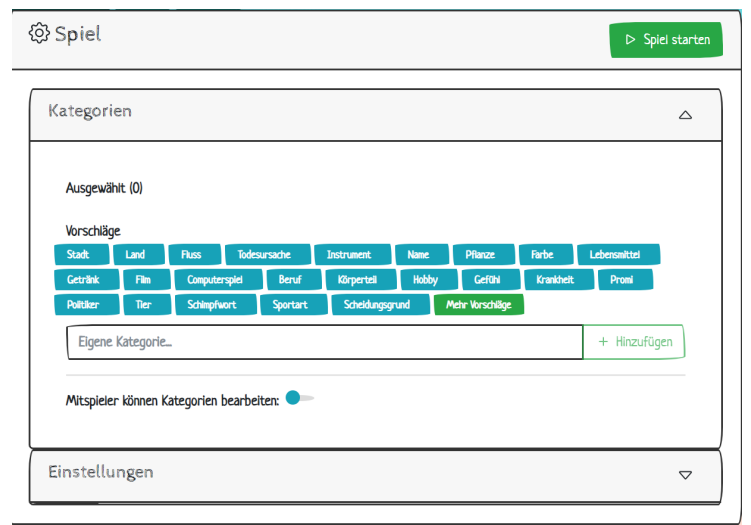

Abb. 3: Kategorien für Stadt-Land-Fluss digital

\section{Arbeit in Kleingruppen}

Kleingruppenarbeit zwischendurch ist ein wichtiges Mittel, um die Studierenden in den Austausch zu bringen, der sonst eher am Kaffeeautomaten stattfindet. Dies ist also nicht unbedingt nur didaktisch hilfreich. Dennoch sollte gut überlegt sein, ob sich die jeweilige Fragestellung für Gruppenarbeiten eignet. Es ist außerdem wichtig, dass man klar kommuniziert, welche Art und Weise der Dokumentation gewünscht ist und wann man wieder zum Hauptraum zurückkehren soll. Die meisten Plattformen bieten zwar die Möglichkeit, die Breakout-Rooms automatisch zu schließen, so dass alle Teilnehmenden direkt zurück zur Veranstaltung gelangen, aber eine Zeitvorgabe und bestenfalls auch ein Hinweis zwischendurch mit der verbleibenden Zeit, helfen den Studierenden, die gemeinsame Arbeit zu strukturieren.

Durch Gruppenarbeiten soll erreicht werden, dass sich alle Beteiligten mit ihren Ideen und ihrem Wissen einbringen können. Dies ist bei den Liberating Structures Zweck aller Methoden. Die Gruppenzusammensetzung ändert sich je nach Methode und Ziel bzw. Fragestellung.

Zur Unterstützung und für die Dokumentation der Ergebnisse sind digitale Whiteboards oder Pinnwände nicht mehr wegzudenken. Tools wie Mural, Miro oder Padlet oder aber auch die jeweils in den Lernplattformen integrierten
Whiteboards sind wichtig, um genau wie im analogen Umfeld festzuhalten, was die Teilnehmenden aus der Gruppenarbeit mitnehmen.

Digitale Whiteboards erzielen eine aus unserer Sicht besonders positive Dynamik, da die vielen Möglichkeiten die Kreativität anregen. Ein gut durchdachter Aufbau des Boards und eine geduldige Einführung in die Anwendung sind essenziell, um die Freude am Mitmachen aller Beteiligten zu gewährleisten. Wir sind überzeugt, dass ein digitales Whiteboard eine hervorragende Basis für Lernreisen sein kann und die $4 \mathrm{~K}$ (Kommunikation, Kreativität, Kollaboration und kritisches Denken) mit Hilfe von Boards gefördert werden können.

\section{Lernreisen auf dem Whiteboard}

Mit digitalen Whiteboards können Lernreisen für Lernveranstaltungen gestaltet werden, in denen die Inhalte und Präsentationen der Dozierenden, passende Videos, Podcasts oder Bücher bzw. Arbeitsflächen mit konkreten Fragestellungen im Board verankert werden. So wird den Studierenden ermöglicht, jederzeit selbständig zu lernen, da die synchronen und asynchronen Lernphasen mit Hilfe eines digitalen Whiteboards geschickt verbunden werden können.

Um zusammenzuarbeiten, wird ein Link zum Whiteboard generiert, der allen Teilnehmenden z.B. über die Chatfunktion des Videokonferenz-Tools zugesandt wird. Die Teilnehmenden können mit diesem Link entweder anonym oder mit Namen auf das Board gelangen und dieses bearbeiten.

Digitale Whiteboards verfügen über zahlreiche Funktionalitäten, die man selbst gut kennen sollte. Obwohl die von der DIU genutzten Tools Miro und Mural grundsätzlich intuitiv aufgebaut sind, ist es ratsam, sich vorab intensiv mit dem gewählten Tool zu befassen und bestenfalls allen Teilnehmenden vorab ein Erklärvideo zukommen zu lassen, damit in der Lernveranstaltung nicht zu viel Zeit für Erläuterungen verloren geht. Erfahrungsgemäß ist die erste Anwendung dennoch immer mit vielen Rückfragen zur Nutzung verbunden. Das ist bei der Konzeptionierung einzuplanen.

Vor allem bei aufwändigen Layouts mit vielen 
verschiedenen Bausteinen empfehlen wir, das Board zunächst z.B. über einen geteilten Bildschirm zu zeigen und zu erklären, bevor der Link veröffentlicht wird.

Digitale Whiteboards können je nach Thema gestaltet werden. Der Kreativität sind kaum Grenzen gesetzt. Es ist ratsam, eine Agenda oder einen Übersichtsplan an den Anfang zu stellen und von dort aus zu den betreffenden Übungen, Aufgaben, Informationen, Empfehlungen etc. im Board zu verlinken, so dass es Nutzer:innen möglichst einfach haben, sich auf dem Board zurechtzufinden. Ein Test vorab ist sinnvoll, damit sichergestellt ist, dass alles fixiert ist, was zur Struktur des Boards gehört und die Verlinkungen im Board funktionieren. Sind viele Teilnehmende auf dem Board kann es sonst durchaus chaotisch werden.

Alle Whiteboard-Tools bieten zahlreiche vorgefertigte Templates an, die die Gestaltung einer Lernreise vereinfachen können. Die Areas und Templates können gespeichert werden, deshalb empfehlen wir, gleichbleibende Basisinhalte zu speichern und dann immer wieder zu verwenden. So spart man wertvolle Zeit und erreicht einen hohen Wiedererkennungseffekt.

Wir haben in unseren Templates immer eine Area für Feedback, Fragen und Empfehlungen vorgesehen, so dass das Miteinander gefördert wird und auch wir uns und unsere Workshops weiterentwickeln können.

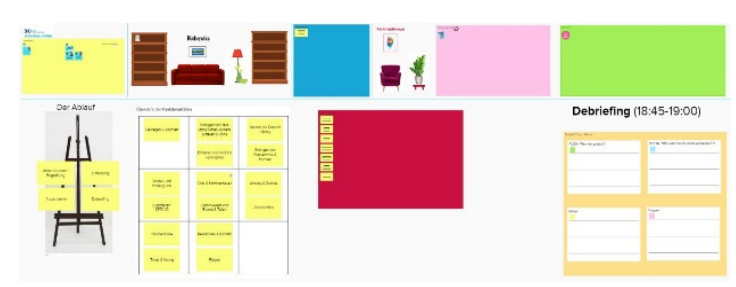

Abb. 4: Ausschnitt aus dem DIU-TeammeetingTemplate (Mural)

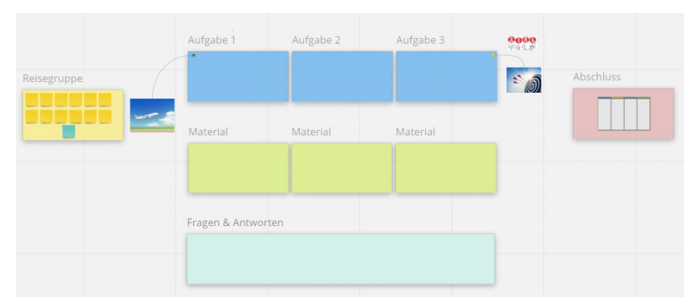

Abb. 5: Lernreise vereinfacht auf einem MiroBoard
Studierende und Lehrende können meist von kostenfreien Education-Lizenzen profitieren.

\section{Selbstorganisiert asynchron lernen}

"Gebt den Lernenden ihr Lernen zurück." (K. Pape, Geschäftsführer der Corporate Learning Community, Slogan in seinem LinkedInProfil)

Ein Teil der Wissensvermittlung sollte asynchron stattfinden z.B. über Lernvideos, die sich die Studierenden selbständig anschauen können. Es ist dabei hilfreich, eine Fragestellung zum Video mitzugeben, die dann wiederum Platz in der synchronen Lehrveranstaltung findet und zum Austausch untereinander führt. Dieses Blended-Learning-Prinzip verzahnt quasi selbstorganisiertes Lernen mit Gemeinsamlernzeit. Dabei ist zunächst völlig unerheblich, ob dies digital oder analog geschieht. Die Digitalisierung erhöht im Grunde nur die Vielfalt der Aufbereitung der Lerninhalte.

Da es unterschiedliche Lerntypen gibt, ist es sinnvoll, Informationen nicht nur als Kopie aus einem Buch, sondern eben auch audiovisuell als Video oder auditiv als Podcast zur Verfügung zu stellen.

Damit einhergehend: Es ist nicht zielführend, ganztägige Lehrveranstaltungen synchron digital durchzuführen. Das Flipped-ClassroomPrinzip ist eine weitaus sinnvollere Methode und ermöglicht, dass Studierende selbstorganisiert lernen können und dennoch immer wieder gemeinsam mit den Dozierenden und Mitstudierenden das Erlernte austauschen und auch auf den Prüfstand stellen können.

Im Austausch mit berufsbegleitenden Studierenden der DIU wurde dennoch klar kommuniziert, dass fest geplante Lernzeit notwendig ist, um neben dem Beruf Platz für Lernen zu schaffen und auch einzuhalten. Hier greift das Prinzip der regelmäßigen gemeinsamen Lernveranstaltungen. In WOL- (Working out loud) oder auch in lernOS-Zirkeln wird ein einstündiges wöchentliches Treffen des Zirkels vereinbart, um sich zu motivieren, auszutauschen und Impulse zu geben. Der Zirkel trifft und unterstützt sich 12 Wochen lang regelmäßig. Mithilfe von Leitfäden mit kleinen Übungen wird ein klarer Rahmen für die Treffen gesetzt. Den Rest der Woche investiert jedes Zirkelmitglied 
so viel Zeit und Energie in die Erreichung des persönlichen Ziels, wie es möchte.

Aufgrund des Erfolgs dieser Zirkel hat sich daraus ein siebenwöchiger Learning-out-loudZyklus entwickelt, den man für seine Lernreise adaptieren kann.

Wir sind überzeugt, dass Lehrende zukünftig zu Lernbegleiter:innen werden, die motivieren, unterstützen, zuhören sowie Impulse und einen Rahmen für das Lernerlebnis ihrer Studierenden geben. Ähnlich wie in den erwähnten Zirkel-Methoden führen die Lernbegleiter:innen die Studierenden immer wieder regelmäBig zusammen und regen den Austausch an und korrigieren wenn notwendig die Richtung der Lernreise. So bekommen Studierende die Freiheit, selbständig ihre Ziele, Inhalte und Methoden zu formulieren und ihre Lernprozesse zu erarbeiten.

\section{Nutzung von MS-Teams}

An der DIU wird hauptsächlich Office365, insbesondere MS-Teams, für Lernveranstaltungen verwendet. Hier sind viele hilfreiche Apps bereits integriert, die es den Dozierenden ermöglichen, die Lernveranstaltung interaktiv zu gestalten und selbstorganisiertes Lernen außerhalb der eigentlichen Lernveranstaltung zu fördern. Die Studierenden und Dozierenden erhalten eine kostenfreie Education-Lizenz.

Mit MS-Teams lassen sich Besprechungen planen mit einer Kalenderfunktion, die mit Outlook synchronisiert ist. In einem Team werden die jeweiligen Studierenden und Dozierenden hinzugefügt und können dort vielfältige Funktionen wie OneNote für Notizen, einen Aufgabenplaner oder ein Umfragetool zum Menü hinzufügen. Es können Beiträge für alle veröffentlicht werden, aber es gibt auch die Möglichkeit, den Chat mit einer oder mehreren Personen zu nutzen. Dateien können ausgetauscht und auch zeitgleich gemeinsam bearbeitet werden - z.B. Word-Dokumente, ExcelTabellen oder Power-Point-Präsentationen.

Von jeder Stelle in MS-Teams heraus können Besprechungen (Videoanrufe) gestartet werden, um Fragen persönlich zu klären.

Natürlich gibt es auch die Möglichkeit, einmal nicht erreichbar zu sein und eine passende Statusmeldung zu hinterlegen.

\section{Hybride Szenarien}

Speziell zu hybridem Lernen haben wir uns viele Gedanken gemacht, da dies zukünftig an der DIU an der Tagesordnung stehen wird.

Mit hybridem Lernen ist ein synchrones Setting gemeint, in welchem einige Personen analog im Seminarraum sind, andere sich digital zuschalten.

Durch die Digitalisierung von Lernveranstaltungen möchten einige Studierende und Dozierende, die vormals eine lange Anreise einplanen mussten, gern im digitalen Raum bleiben. Auch im Krankheitsfalle ist das zeitweise Zuschalten und zumindest Zuhören eine gute Option für Studierende. Andere Teilnehmende freuen sich sehr darauf, sich persönlich im Seminarraum sehen und austauschen zu können. Für beide Gruppierungen möchten wir ein angenehmes und gehaltvolles Setting bieten.

Gute hybride Formate sollten so gestaltet sein, dass alle Teilnehmenden - ob sie nun vor Ort sind oder sich digital zuschalten - gleichermaßen ins Unterrichtsgeschehen einzubeziehen sind. Aus unserer Sicht führt hier kein Weg daran vorbei, digitale Hilfsmittel und Tools in das ursprünglich rein analoge Geschehen einzubetten. Hierfür ist es ratsam, dass die Studierenden vor Ort ebenfalls über ein Endgerät verfügen, mit welchem z.B. Live-Umfragen digital durchgeführt werden können. Meist genügt ein Smartphone. Bei Gruppenarbeiten, in denen digitale und analoge Teilnehmende gemischt werden sollen, ist es notwendig, dass jede Gruppe zumindest ein Notebook oder Tablet (mit Kamera und Mikrofon) zur Verfügung hat. Statt der Tafel kann ein Tablet mit Stift verwendet werden, so dass sowohl die Teilnehmenden vor Ort als auch die digital zugeschalteten Studierenden sehen können, was geschrieben oder gezeichnet wird. Dieses Umdenken und die daraus resultierende Neukonzeptionierung des Lerngeschehens sind zunächst aufwändig, aber notwendig.

\section{Literatur}

[1] Adam, Björn; Holle, Judith; Köpnick, Franziska: Das Methodenbuch für digitalen Unterricht. Dein Praxisbegleiter für gute digitale Lernräume, 1. Auflage, Lüneburg 2021 
Links zu Tools und Methoden: Überblick

http://tscheck.in/

https://internetquatsch.de/

https://liberatingstructures.de/

https://www.mentimeter.com/

https://invote.de/

https://kahoot.it/

https://jeopardylabs.com/

https://skribbl.io/

https://stadtlandfluss.cool/

https://www.mural.co/

https://miro.com/

https://de.padlet.com/

https://cogneon.github.io/lernos/

https://workingoutloud.com/

https://learningoutloud.de/

https://www.microsoft.com/de-de/microsoft-teams 